\title{
On the Possibilities of Decreasing Power Loss in Large Tilting Pad Thrust Bearings
}

\author{
Michal Wasilczuk and Grzegorz Rotta \\ Faculty of Mechanical Engineering, Gdansk University of Technology, Narutowicza 11/12, 80-233 Gdansk, Poland \\ Correspondence should be addressed to Michal Wasilczuk; mwasilcz@pg.gda.pl
}

Received 6 November 2012; Accepted 24 November 2012

Academic Editors: N. Gerolymos and Z. Wen

Copyright (C) 2013 M. Wasilczuk and G. Rotta. This is an open access article distributed under the Creative Commons Attribution License, which permits unrestricted use, distribution, and reproduction in any medium, provided the original work is properly cited.

Different systems of direct oil supply have been developed in order to facilitate efficient introduction of fresh lubricant to the oil gap and reduction of churning power loss in tilting pad thrust bearings. Up to now there is no documented application of the supply groove in large thrust bearings used in water power plants. The results of modeling lubricant flow in the lubricating groove of a thrust bearing pad will be presented in the paper. CFD software was used to carry out fluid film calculations. Such analysis makes it possible to modify groove geometry and other parameters and to study their influence on bearing performance. According to the results a remarkable decrease in total power loss due to avoiding churning losses can be observed in the bearing.

\section{Introduction}

Basic operation limit for any hydrodynamic bearing is minimum film thickness [1]. This limit, called hydrodynamic limit, is especially important in low-speed bearings, for example, hydrogenerator bearings, in which in most cases sliding speed is less than $25 \mathrm{~m} / \mathrm{s}$, and specific loads higher than $2.5 \mathrm{MPa}$. In the same time quite often temperatures of the sliding surface exceed $100^{\circ} \mathrm{C}$. Decreasing bearing temperature causes increasing margin of safety of bearing operation by higher oil viscosity, so at lower temperature, hydrodynamic action is enhanced. Ettles [2] showed that bearing temperature is strongly affected by oil temperature at the inlet to the fluid film and by the runner temperature. Moreover, temperature level in thrust bearings depends on the method of supplying cold oil to the bearing. A series of papers from early 1970s [3-5] indicate that directed lubrication (e.g., spray or groove systems) with evacuated bearing housing is an efficient method of decreasing temperature in high-speed bearings and significant reduction of churning loss. On the other hand for low-speed applications, the authors of the above mentioned papers did not see potential for substantial decrease of bearing temperature.

The most common method of lubricating large thrust bearings is flooded lubrication, in which all the bearing elements are immersed in oil contained in the bearing housing. Quite often flooded lubrication is assisted by special direct oil supply systems. In spite of the fact that the use of such systems have become frequent, their actual influence on bearing characteristics is still unknown, at least in the literature known to the authors of this paper such results have not been published. Decrease of friction losses also becomes an important parameter in assessing bearing performance and the lubrication method has an influence on bearing losses, but, due to difficulty in carrying out experiments, has not been systematically studied in large thrust bearings.

Nowadays computational fluid dynamics (CFD) systems can be used to analyze bearing problems, regardless of dimensions and operating conditions. A numerical model of a large thrust bearing with a supply groove (SG) machined in the inlet area of the bearing pad was elaborated [6-9]. The results of calculations were compared to the results of similar calculations for a bearing with traditional flooded lubrication.

\section{Description of the Model}

A large thrust bearing of a water turbine was studied. Technical and operational data of the analysed thrust bearing are presented in Table 1. 
TABLE 1: Bearing data.

\begin{tabular}{lccc}
\hline & & Unit & Value \\
\hline Outer diameter & $D_{\text {out }}$ & $\mathrm{m}$ & 1.78 \\
Inner diameter & $D_{\text {in }}$ & $\mathrm{m}$ & 0.814 \\
Pad thickness & $G$ & $\mathrm{~m}$ & 0.19 \\
Number of pads & - & - & 8 \\
Rotational speed & $n$ & $\mathrm{rpm}$ & 214 \\
Oil supply flow rate & $\mathrm{Q}$ & $\mathrm{dm}^{3} / \mathrm{min}$ & 330 \\
Oil type: ISO VG46 & & & \\
$\quad$ Oil viscosity at $T=40\left({ }^{\circ} \mathrm{C}\right)$ & $\mu_{\text {nom }}$ & $\mathrm{Pa} \cdot \mathrm{s}$ & 0.039 \\
$\quad$ Oil density & $\rho$ & $\mathrm{kg} / \mathrm{m}^{3}$ & 852 \\
$\quad$ Oil specific heat & $c_{\mathrm{ol}}$ & $\mathrm{J} / \mathrm{kg} \cdot \mathrm{K}$ & 2000 \\
$\quad$ Oil heat conductivity & $k_{\mathrm{ol}}$ & $\mathrm{W} / \mathrm{m} \cdot \mathrm{K}$ & 0.13 \\
Reference temperature & $T_{\text {nom }}$ & ${ }^{\circ} \mathrm{C}$ & 40 \\
\hline
\end{tabular}

Figures 1 and 2 show geometrical models used for the numerical simulations. Fluid part of the model consists of fluid film, region below pad, and space between pads. The space between pads is divided into two parts-one adjacent to the pad upstream (inlet) part and the other to the downstream (trailing) part. Between these two parts a periodic boundary condition was set to simulate a whole bearing, which is a sequence of several pads with the outlet from one being the inlet to the next. In this way, a model is a continuity as a bearing consisting of several pads-flow, temperatures, and energy transportation occurs from pad outlet to inlet just as it occurs in a real bearing between its pads.

There were two models: one for a bearing with flooded lubrication and the other one for a bearing with a supply groove. Main difference between these two models was the location of cold oil inlet. It was situated in selected nodes at the bottom of the bearing housing in case of flooded lubrication and in the supply pipe in the lubrication groove in case of groove lubrication. The whole fluid model for flooded lubrication consisted of 130000 elements, while the supply groove lubrication model was divided into 166000 elements. The number of elements was determined on the basis of calculations in which influence of the mesh size on the results was tested [10]. The difference in the number of elements is due to existence of mesh grid in lubricated groove. Fluid film part including inlet and outlet chamfers has exactly the same mesh in both cases. Time required for reaching convergence was about 12 hours. Specification of the workstation used for calculation: 1 CPU AMD Dual-Core Opteron 275 (64 bit, 2.2 GHz), 4 GB RAM. Fast SCSI hard drive was used as the data storage.

Empty space inside the fluid part of the model (Figures 1 and 2) is filled with the structural part of the model, that is, bearing pad. Above the fluid film, the runner is located which is an element of the structural part of the model, as well.

In order to compare load carrying capacity of the bearings in all numerical simulations fluid film had constant minimum film thickness of $25 \mu \mathrm{m}$. It is a well-known fact that thermoelastic deformations have a great influence on performance of large thrust bearings. In order to include these deformations without adding too much complexity to the calculations, the deformations were calculated in the external bearing model. This model is a combination of FEM (finite element method) structural model and THD (thermohydrodynamic) finite difference model of the film and it was described in detail in [11]. Fluid film geometry shown in Figure 3 is the result of such calculations performed for the data of the bearing being studied in this paper. In the contour map, one can see film thickness expressed in micrometers with the inlet at the left-hand side. The position of minimum film thickness of $25 \mu \mathrm{m}$ is located $89 \%$ of the pad length circumferentially from the inlet and $62 \%$ of the pad width radially from the inner radius. Crowning of the pad in radial cross section can be evaluated as approximately $50 \mu \mathrm{m}$.

Due to the constant film thickness load capacity of the bearing and maximum film temperature were the main representative results describing efficiency of the supply groove.

Calculations described in this paper were made with the use of ANSYS Multi-Field Solver (MFX) which is primarily intended for FSI which combines two programs: ANSYS CFX for fluid calculations and ANSYS Mechanical for structural calculations. This procedure assumes that heat conduction and temperature field distribution are calculated in a structural model and boundary temperatures are then transferred to the fluid model as boundary conditions. After the run of a fluid solver heat fluxes with profile preservation (value and distribution) are applied to the structural model as boundary conditions. Fluid film calculations were performed according to the model implemented in ANSYS CFX. Fluid grid is made of 8-node element and task is solved with the use of Reynolds Averaged Navier-Stokes (RANS) equations with the use of Finite Volume Method (FVM) as follows:

$$
\begin{gathered}
\frac{\partial \rho}{\partial t}+\nabla \bullet(\rho U)=0 \\
\frac{\partial \rho U}{\partial t}+\nabla \bullet(\rho U \otimes U)=\nabla \bullet(\tau-\rho \overline{u \otimes u})+S_{M} \\
\frac{\partial \rho h_{\text {tot }}}{\partial t}-\frac{\partial p}{\partial t}+\nabla \bullet\left(\rho U h_{\text {tot }}\right) \\
=\nabla \bullet(\lambda \nabla \tau-\rho \overline{u h})+(U \bullet \tau)+S_{E}
\end{gathered}
$$

For solving these equations, an Algebraic Multigrid technique is used within the solver of the software used for the described calculations. It is an accelerated Incomplete Lower Upper factorization technique that solves discrete system of linearized equations. Algebraic Multigrid forms a system of discrete equations for a coarse mesh by summing the fine mesh equations. This results in virtual coarsening of the mesh spacing during the course of the iterations, and then rerefining the mesh to obtain an accurate solution. A particular implementation of Algebraic Multigrid called Additive Correction is used in ANSYS CFX [12].

During thermal calculations in ANSYS Mechanical SOLID90 element type were used. It is a 3D, 20-node thermal element, which is used in analysis of thermal conduction problems only [12]. Meshes of both models do not have to 


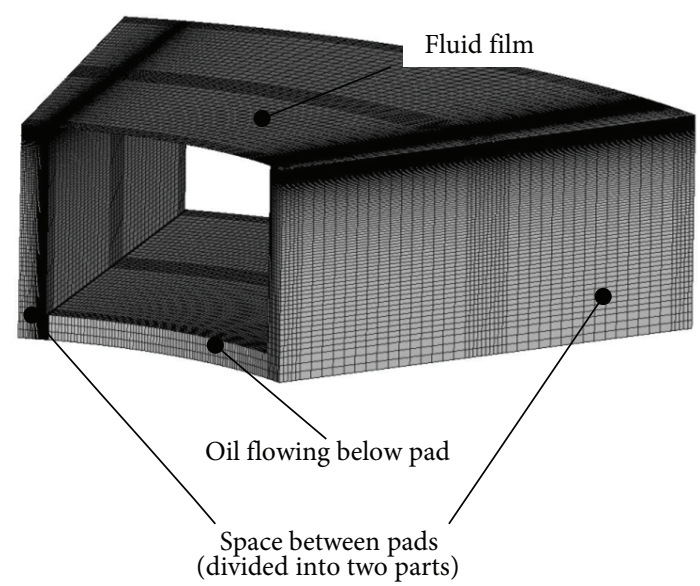

FIGURE 1: General geometrical model of the fluid part surrounding the bearings pad (real mesh grid used in calculations).

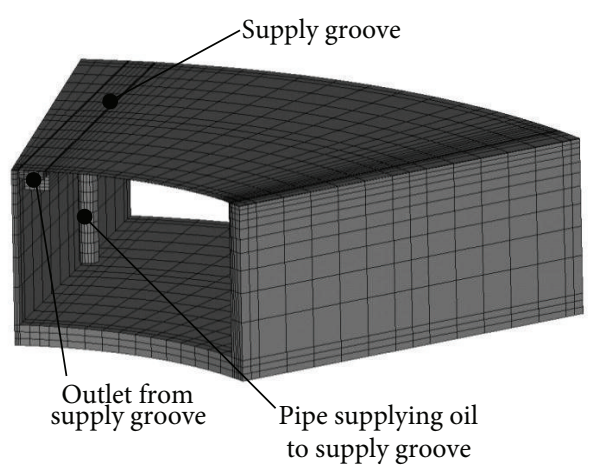

FIGURE 2: General geometrical model of the fluid part surrounding the bearings pad with lubrication groove (coarse mesh grid used for better clarity of the picture).

correspond to each other due to interpolation procedures offered by the software. They may have different mesh densities so each can be adjusted to the requirements of the model. It is only necessary to assure that the meshes geometrically complement each other.

Fluid model consists of both hydrodynamic oil film and oil bath surrounding the bearing pad on all the remaining walls apart from inner and outer periphery, which are not surrounded by the fluid model (see Figures 1 and 2)-on these peripheries heat convection coefficient of $\alpha=$ $400 \mathrm{~W} / \mathrm{m}^{2} \mathrm{~K}$ was assumed on outer and inner pad periphery on the basis of [13]. At all other solid-fluid boundaries, no additional assumptions are necessary since boundary conditions are calculated and updated during simulation. External model boundaries such as bottom of the housing and upper part of the runner were treated as adiabatic. Steady state conditions and incompressible fluid were assumed. Laminar or turbulent flow was automatically established due to software special turbulence modeling.

Turbulence model $k-\omega$ based SST (Shear Stress Transport) was used, and it belongs to two-equation turbulence models. Effect of using this model is that laminar regions (e.g.,

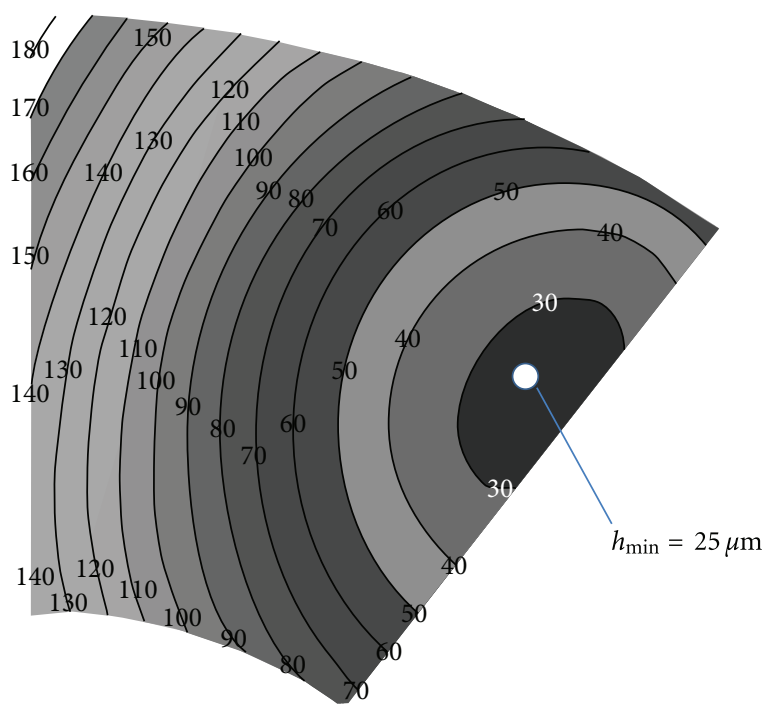

FIGURE 3: Fluid film shape used in calculations-contours show film thickness in $[\mu \mathrm{m}]$. Inlet at the left-hand side, minimum is located $89 \%$ circumferentially from the inlet and $62 \%$ radially from the inner radius.

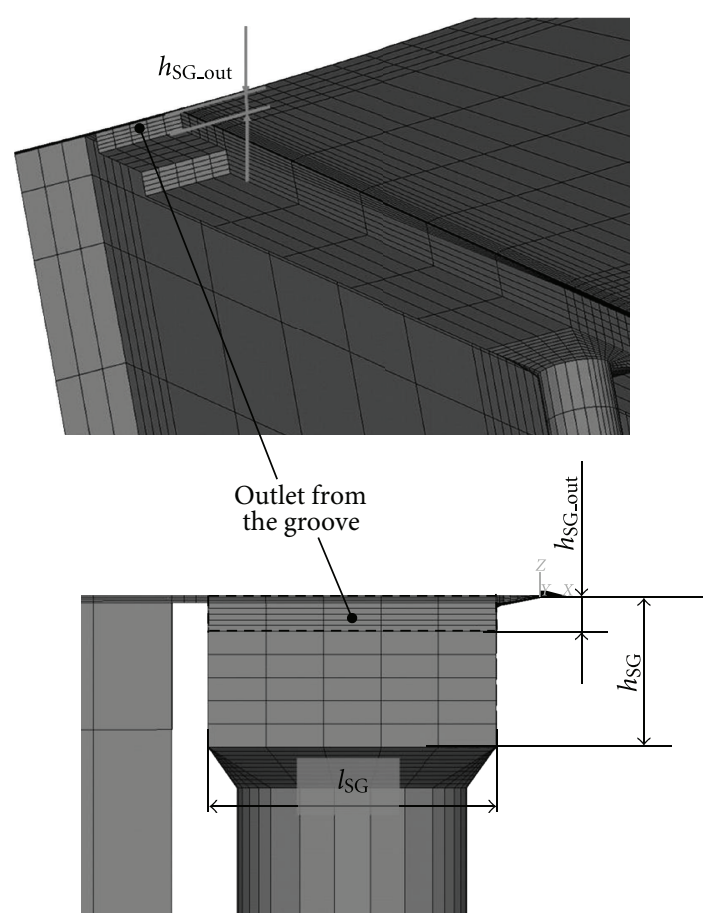

FIGURE 4: Details of the supply groove.

thin oil film) are automatically treated as a laminar flow while turbulence regions (e.g., space between pads, supply groove) have turbulent flow regime due to consideration of turbulence transition.

Viscosity is modeled as a function of the local temperature with the use of Sunderland formula [12], due to relatively low pressure in the hydrodynamic film, the influence of pressure 


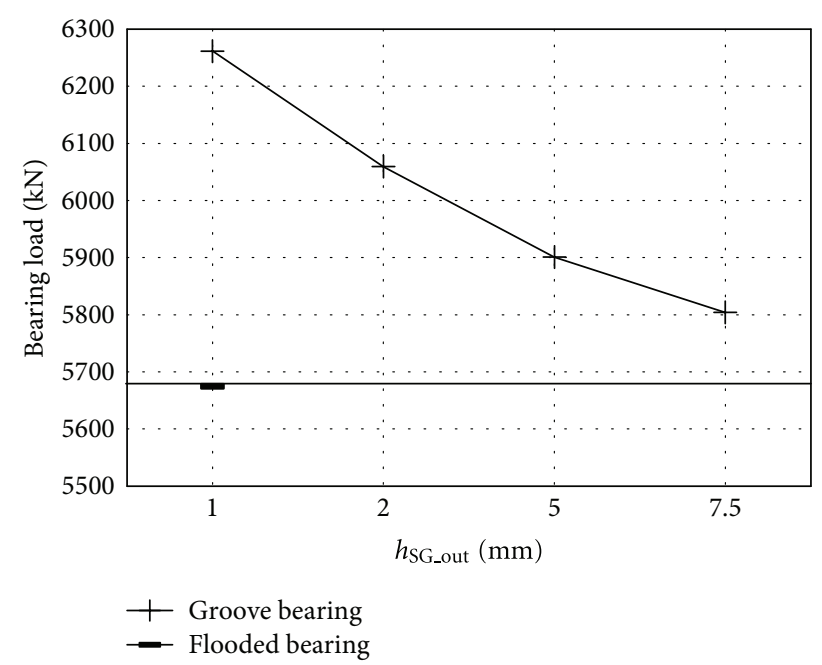

FIGURE 5: Bearing load carrying capacity versus height of the supply groove outlet $\left(h_{\mathrm{SG} \_ \text {out }}\right)$.

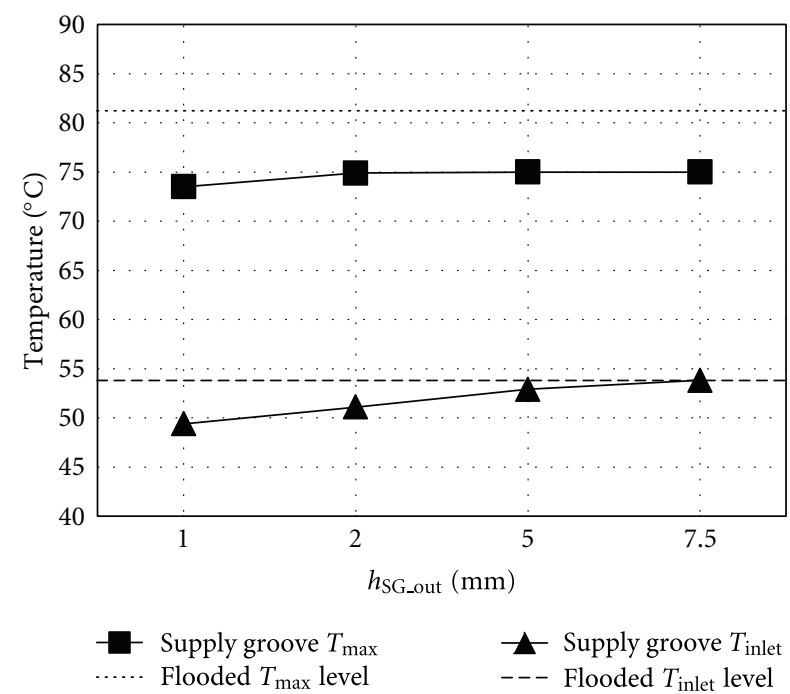

FIGURE 6: Temperature versus height of the supply groove outlet $\left(h_{\text {SG_out }}\right)$.

on viscosity was neglected, which is a standard practice in hydrodynamic analysis

$$
\mu(T)=\mu_{N} \cdot e^{\left(C_{2}\left(1 / T-1 / C_{1}\right)+C_{3}\left(1 / T-1 / C_{1}\right)^{2}\right)},
$$

where $\mu(T)$ is the oil viscosity $[\mathrm{Pa} \cdot s] ; T$ is the temperature $[\mathrm{K}] ; \mu_{N}$ is the nominal viscosity $[\mathrm{Pa} \cdot \mathrm{s}] ; C_{1}$ is the first viscosity coefficient, equal to absolute temperature at which $\mu=\mu_{N}$ where $C_{1}=313.16 ; C_{2}$ is the second viscosity coefficient where $C_{2}=3140.832 ; C_{3}$ is the third viscosity coefficient where $C_{3}=-946242.894$.

\section{Numerical Results and Discussion}

Numerical solutions for different dimensions of the outlet from the supply groove $\left(h_{\mathrm{SG} \_o u t}\right.$ parameter in Figure 4$)$ were obtained and they were compared to those of the bearing with flooded lubrication (Figure 1).

Axial load $F$, maximum film temperature $T_{\max }$, and average inlet temperature $T_{\text {inlet }}$ were compared as parameters demonstrating lubrication system efficiency.

The results are presented in Figures 5 and 6. The results show (Figure 5) that by using a lubrication groove load carrying capacity can be increased by up to $10 \%$, that is, by almost $600 \mathrm{kN}$ in comparison to the bearing with traditional flooded lubrication (assuming the same minimum film thickness). At the same time despite higher bearing load, maximum bearing temperature was still lower by almost $8^{\circ} \mathrm{C}$ (Figure 6).

Influence of some other geometrical parameters was also studied, and changes in the height of the groove side outlet window (" $h_{\text {SG_out }}$ ") had a considerable effect on bearing load $F$ (Figure 5) which was decreased by $480 \mathrm{kN}$ for the largest value of $h_{\text {SG_out }}$, maximum bearing temperature changed only by $1.5^{\circ} \mathrm{C}$ (Figure 6). Other studied parameters like for example groove depth $\left(h_{\mathrm{SG}}=5 \div 15 \mathrm{~mm}\right)$ had almost no influence on bearing characteristics - change of load capacity was less than $25 \mathrm{kN}$ and in maximum temperature less than $0.1^{\circ} \mathrm{C}$. In all cases considered the same flow rate of cold oil was used.

Temperature map of the pad sliding surface for both lubrication methods is shown in Figure 7. One can observe higher efficiency in introducing cold oil directly to the fluid film by means of supply groove method, demonstrated by larger area of the sliding surface with temperature lower than $55^{\circ} \mathrm{C}$ in case of groove lubrication (Figure $7(\mathrm{~b})$ ).

Velocity field in the bearing with the supply groove shown as streamlines of oil flowing from selected points of supply hole is shown in Figure 8. According to the image shown in Figure 8(a) most of the cold oil moves across lubrication groove and only small part of fresh oil enters directly the fluid film. In Figure 8(b), one can see whirling of oil during its flow to the outlet. It is probably an explanation for higher efficiency of the supply groove reported in [14]: efficient heat exchange in lubrication groove and in the nearest vicinity of the inlet to the pad is due to forced convection (whirling of oil) and results in efficient decreasing of inlet temperatures.

Temperature field at the inlet to the fluid film is shown in Figure 9. Cross-sections in Figures 9(b), 9(c), and 9(d) are made at the inlet to the film and shown in the direction of motion, outer bearing diameter is at the left hand side. Lower oil temperature close to the outer diameter in Figure 9(b) (flooded lubrication) is caused by pumping effect of the collar. It means that cold oil, which is introduced in the middle part of the space between pads, is pumped towards the outer diameter of the bearing due to centrifugal effect. In case of the supply groove (Figures 9(c), and 9(d)) lower temperatures near the inner diameter are caused by the outflow of cold oil excess from the groove through the outlet situated at the inner bearing diameter.

\section{Decreasing of Churning Power Loss}

As it was mentioned by Martin [3], Bielec and Leopard [4], Mikula and Gregory [14], and New [15], one of the 


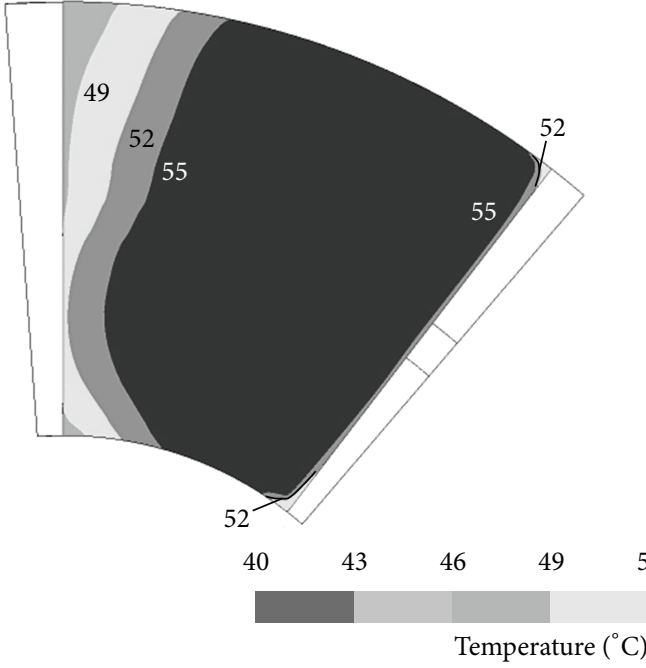

(a)

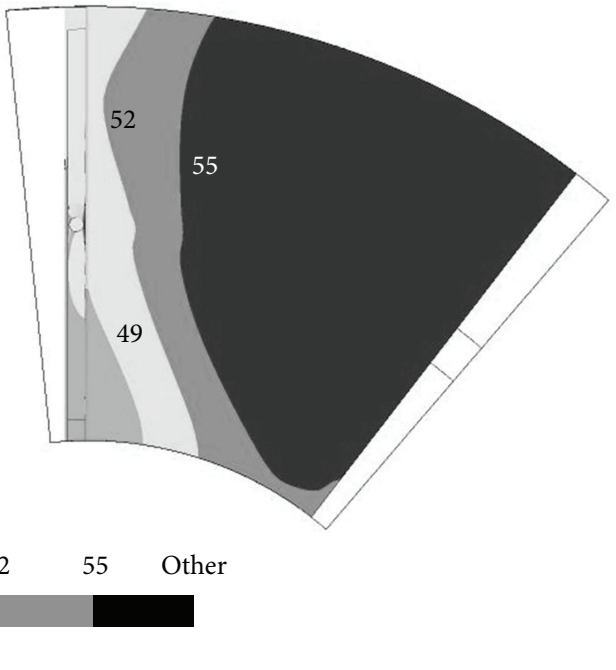

(b)

FIGURE 7: Temperature profile on pads sliding surface: (a) flood lubricated bearing, (b) groove lubricated bearing.

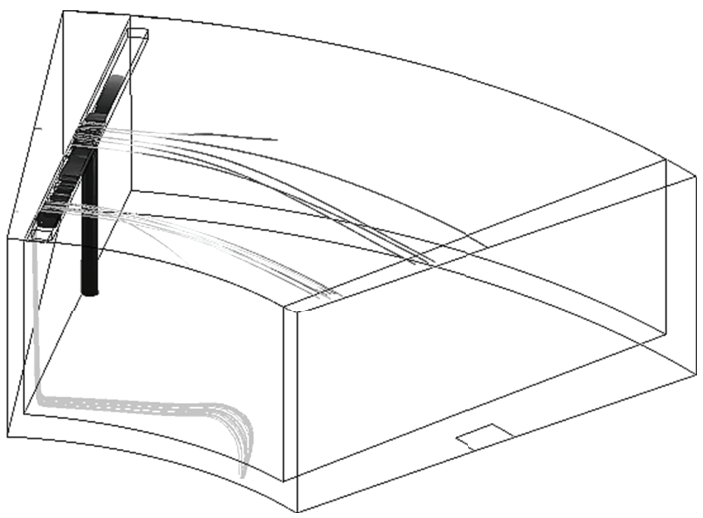

(a)

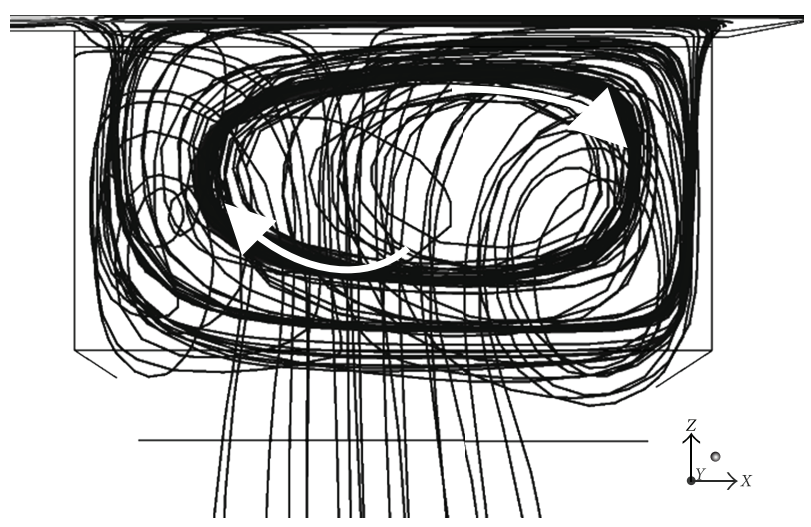

(b)

FIGURE 8: Oil particle flow traces in the bearing with supply groove, (a) general view, (b) whirling of oil in the groove.

potential benefits of using directed lubrication is the decrease of power loses caused by churning of oil in the bath. Directed lubrication is realized by removing all the oil from the bearing housing, while the amount of cold oil required for hydrodynamic lubrication is supplied directly to the inlet zone of the bearing pads. Proportions of different sources of power loss in a thrust bearing are shown in Figure 10. In an example of high-speed bearing taken from [3-5] (OD = $0.125 \mathrm{~m}, n=12000 \mathrm{rpm} \rightarrow v=60 \mathrm{~m} / \mathrm{s})$ churning losses are about $46 \%$ of total power loss (line described as "1" in Figure 10). On the other hand, in a large thrust bearing, like the one analysed in this paper (OD $=1.78 \mathrm{~m}, n=214 \mathrm{rpm}$ $\rightarrow v=14.5 \mathrm{~m} / \mathrm{s}$ ), churning losses can be estimated as $15 \%$ of total power loss (line "2" in Figure 10).

If one realizes that some contemporary large thrust bearings, especially in pumped storage power plants, operate with sliding speed exceeding $30 \mathrm{~m} / \mathrm{s}$ potential, decrease of churning losses due the use of supply grooves seems to be encouraging.
On the other hand, with the use of supply grooves and evacuated bearing housing, there is a risk of bearing damage in case of a failure of electrical or mechanical part of a supply system, as lack of lubrication will occur in this situation. This, however, would not happen in the case of flooded lubrication, and the bearing will operate properly in hydrodynamic lubrication regime. Ability to operate for several minutes without external cooling of oil is quite a common contract requirement for large thrust bearings used in water turbines. In case of circulating pump failure, such a requirement is quite justified.

4.1. Assumptions for Power Loss Calculations. An extended bearing model (267 000 elements, computing time c.a. 18 hours) allows to estimate power loss due to churning of oil in the oil bath. Main components of the churning loss are at the inner and outer diameter of the collar rim and at the parts of the runner which are above the space between pads (Figure $11)$. 


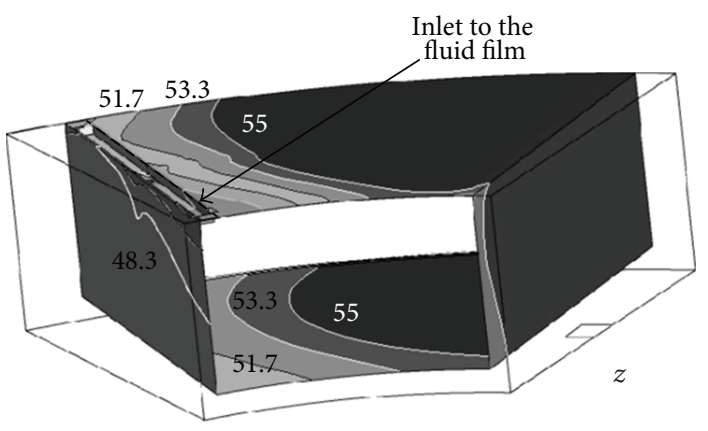

(a)

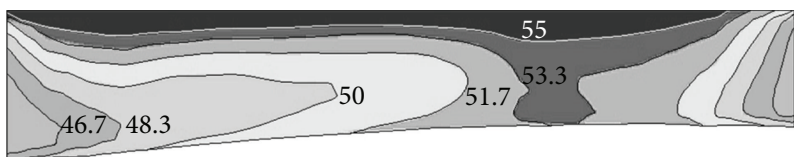

(b)

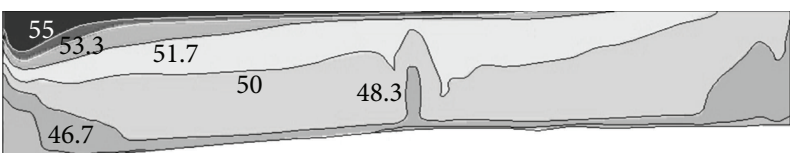

(c)

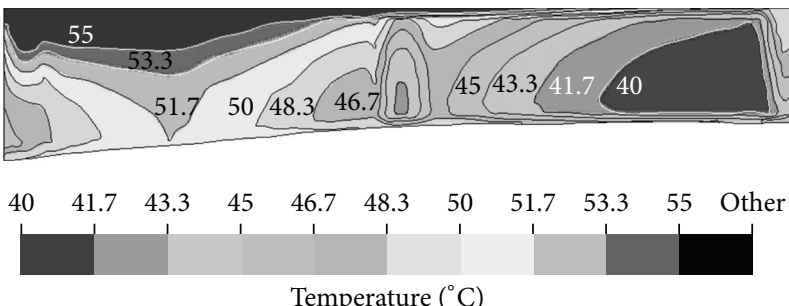

(d)

FiguRE 9: Temperature profile on the inlet to the fluid film (film thickness enlarged 500x): (a) general view, (b) flooded lubrication $T_{\text {inlet }}=$ $53.5^{\circ} \mathrm{C}$, (c) groove lubrication, $h_{\mathrm{SG}_{\text {_out }}}=5 \mathrm{~mm}, T_{\text {inlet }}=52.9^{\circ} \mathrm{C}$, (d) groove lubrication, $h_{\mathrm{SG}_{\text {oout }}}=1 \mathrm{~mm}, T_{\text {inlet }}=49.4^{\circ} \mathrm{C}$.

TABLE 2: Comparison of power loss at the collar rim according to height of the collar immersion.

\begin{tabular}{lccc}
\hline Component of power loss & \multicolumn{2}{c}{$\begin{array}{c}\text { Percentage of total power loss for various cases } \\
\text { Large bearing }\end{array}$} & $\begin{array}{c}\text { Medium size bearing, medium speed } \\
\text { Medium size bearing, high speed }\end{array}$ \\
\hline$P_{\text {collar_in }}$ & $0.2 \%$ & $6.4 \%$ & $2.5 \%$ \\
$P_{\text {collar_out }}$ & $3.4 \%$ & Small immersion & $12.3 \%$ \\
\hline$P_{\text {collar_in }}$ & $0.1 \%$ & $3.7 \%$ & $1.5 \%$ \\
$P_{\text {collar_out }}$ & $1.1 \%$ & Decrease of total power loss due to decreasing of collar immersion & $6.8 \%$ \\
\hline & & $3.6 \%$ & $6.5 \%$ \\
\hline
\end{tabular}

Main component parts of power losses take place at

(i) runner in thin film area $\left(P_{r_{-}}\right.$film $)$,

(ii) runner over the groove between pads $\left(P_{r_{-} \text {groove }}\right)$,

(iii) inner collar diameter $\left(P_{\text {collar_in }}\right)$, (iv) outer collar diameter $\left(P_{\text {collar_out }}\right)$. $\left(P_{s}\right)$

All of them add to generate a total power loss on the shaft

$$
P_{s}=P_{r_{-} \text {film }}+P_{r_{-} \text {groove }}+P_{\text {collar_in }}+P_{\text {collar_out }}
$$




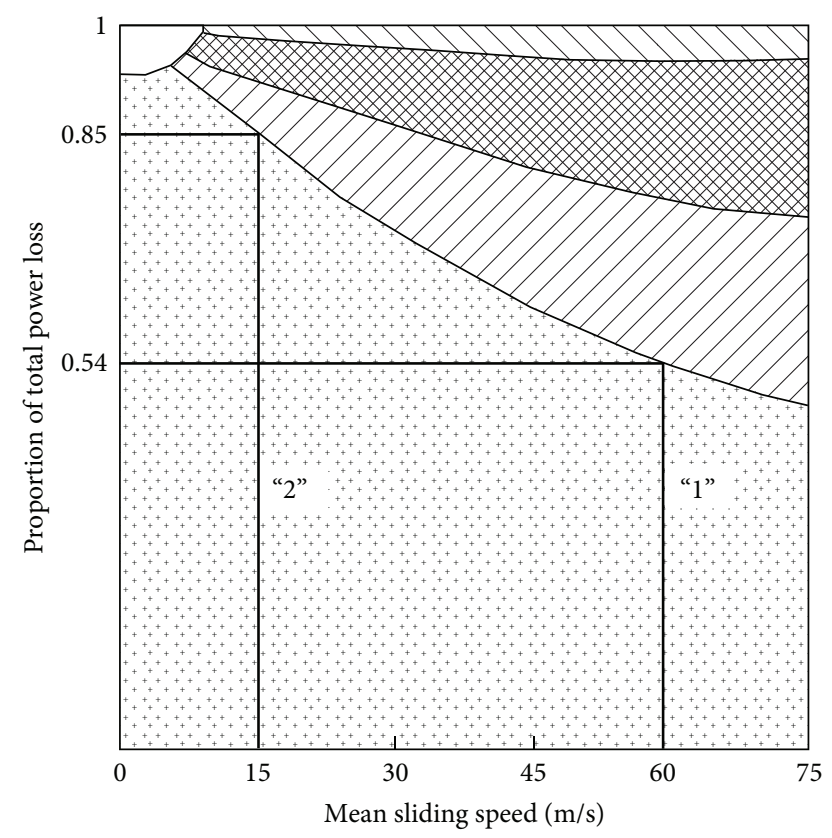

Dhurning losses on pad inner diameter and shaft

Churning losses at rim of collar

$7 A$ Churning losses between pads

Churning losses on surge and main face pads

Figure 10: Power loss in a thrust bearing in case of flooded lubrication [3].
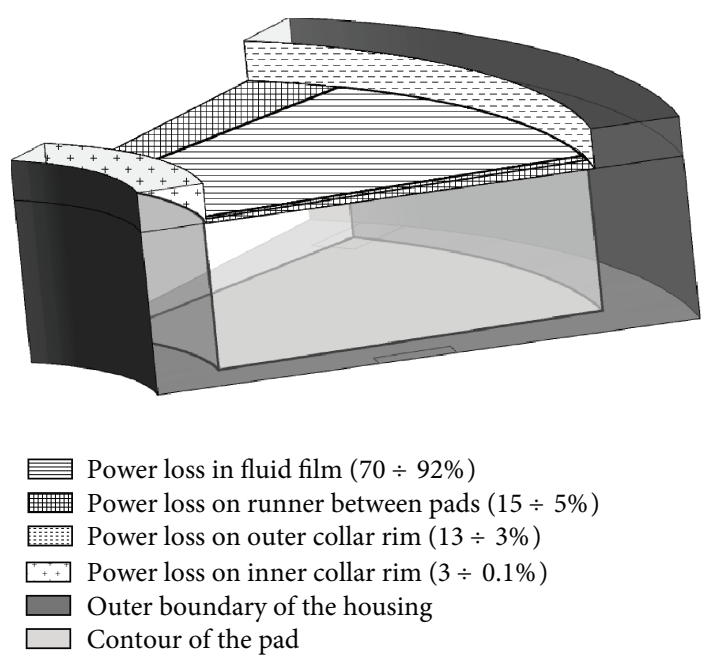

FIGURE 11: Components of power loss in flood lubricated bearing. Values in the brackets are approximate, and they are obtained in the current numerical analysis, for different types of bearings and operational conditions.

while both runner components make a total runner power loss $\left(P_{r}\right)$ as

$$
P_{r}=P_{r_{-} \text {film }}+P_{r_{-} \text {groove }}
$$

power loss due to friction in thin fluid film $\left(P_{r_{\text {ffilm }}}\right)$ cannot be avoided in a hydrodynamic bearing while other components like $P_{r_{-} \text {groove }}, P_{\text {collar_in }}$, and $P_{\text {collar_out }}$ can be partially or even completely avoided. The following problems have been analyzed in relation to churning power loss:

(i) influence of bearing dimensions and sliding speed,

(ii) influence of rate of immersion of the shafts sides,

4.2. Influence of Bearing Dimensions and Sliding Speed. Bearing dimensions and sliding speed are the most important factors influencing churning loses. Sliding speed is strongly affected by bearing dimensions and rotational speed. To assess this influence, three cases were studied: low-speed large thrust bearing $\left(D_{m}=1.297 \mathrm{~m}, n=214 \mathrm{rpm}(v=14.53 \mathrm{~m} / \mathrm{s})\right.$ and medium size thrust bearing $\left(D_{m}=0.6096 \mathrm{~m}\right)$ with medium $(n=600 \mathrm{rpm}, v=19.15 \mathrm{~m} / \mathrm{s})$ and high sliding speed $(n=1200 \mathrm{rpm}, v=38.3 \mathrm{~m} / \mathrm{s})$. The results of calculations for these three cases are shown in Figure 12. The results show that in a large bearing almost $10 \%$ of the losses can be attributed to the avoidable part (other than film) as compared to even $30 \%$ for a high-speed bearing. If one considers only the avoidable losses, it can be observed that in all three cases about half of the avoidable power loss is generated in the groove-part of the runner and the remainder on the outer rim of the collar. Calculated share of the avoidable power is slightly lower than shown in Figure 10, nevertheless, it is still considerable.

\section{Influence of Immersion Depth of the Collar Sides}

This comparison was made for two cases of submerging collar sides (Figure 13) and indicates that churning power loss on inner and outer surfaces of the shaft changes with changes of immersion rate. Results shown in Table 2 were obtained for $50 \mathrm{~mm}$ submerged thrust collar in a large bearing and 30 $\mathrm{mm}$ for a medium bearing. As it is seen in Table 2, depending on the sliding speed and bearing dimensions, it is possible to decrease this power loss by over $7 \%$, only by reducing oil quantity in the bath to the level slightly over the sliding surface of the shaft. Disadvantage is that the larger the bearing the smaller the gain from decreasing shaft immersion and for large low-speed bearing the gain is only $2.5 \%$.

It should be noticed that in case of bearing with supply groove at the leading edge, oil will be delivered directly to the inlet into the fluid film, which prevents oil starvation. Probably it will require additional action to maintain constant oil supply to the film, for example, dual oil pumping system, in order to avoid the risk of failure as discussed above.

\section{Conclusions}

Presented results show that supply groove at the leading edge of the pad has a potential for improving running characteristics of large thrust bearings, without any increase in cold oil flow. Supply pressure in the lubrication groove in all cases was observed to be less than $0.1 \mathrm{MPa}$, which shows that the increase in load carrying capacity is not an effect of hydrostatic action of the supply groove. 


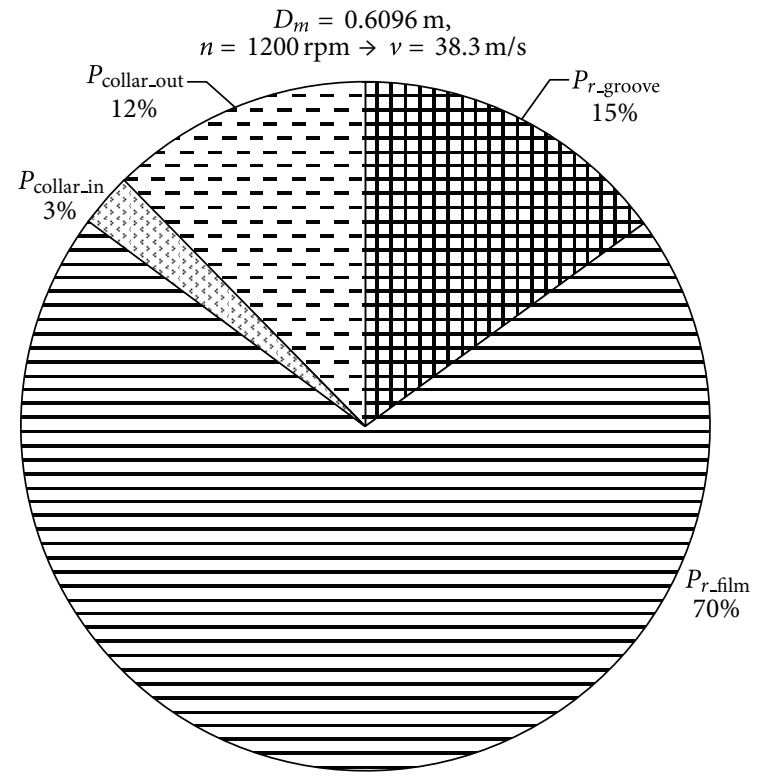

(a)

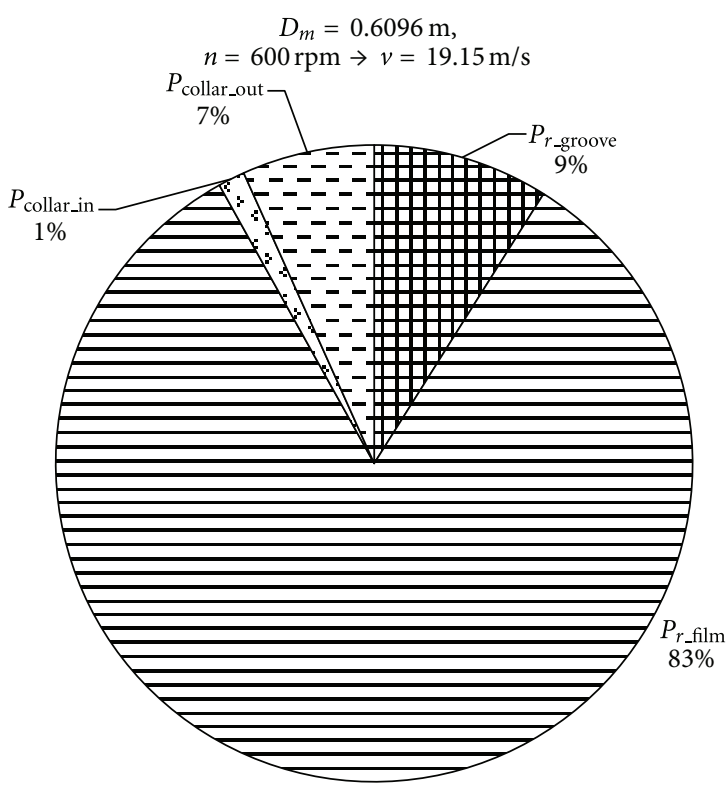

(b)

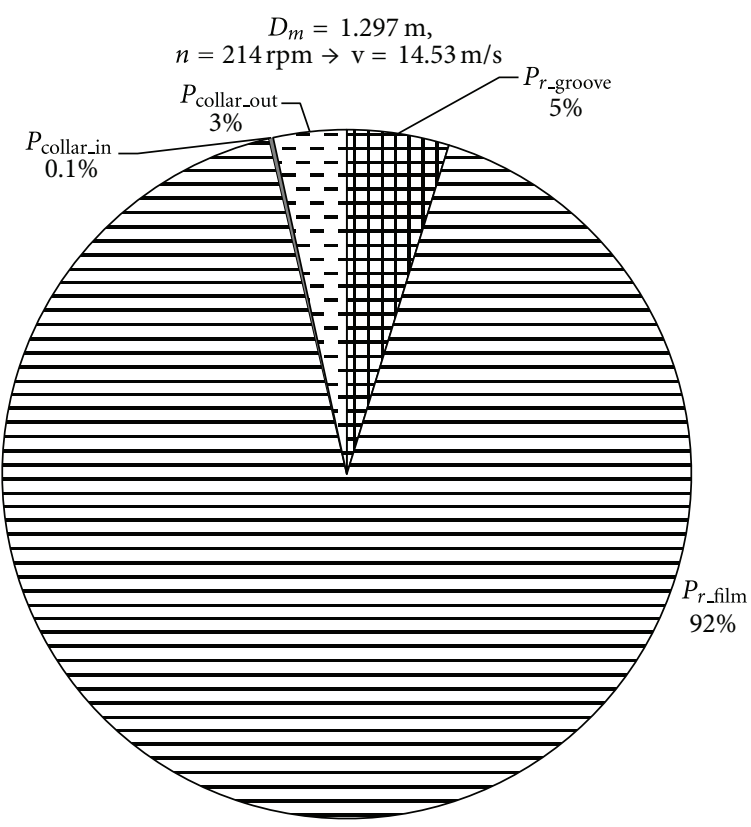

(c)

FIGURE 12: Comparison of power loss in three regimes of bearing operations depending on size and rotational speed.

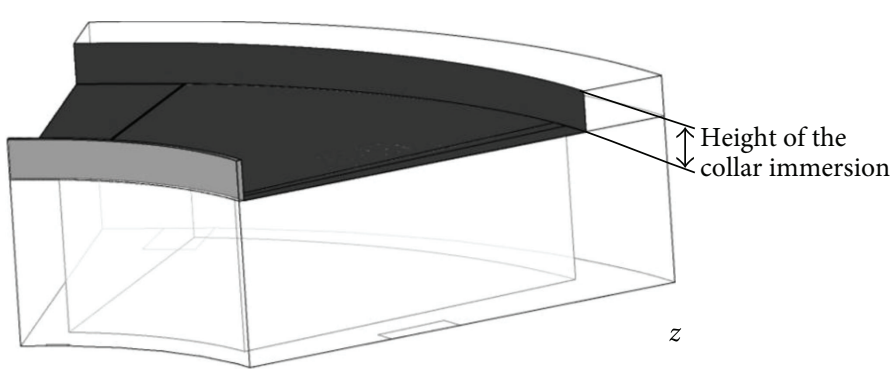

(a)

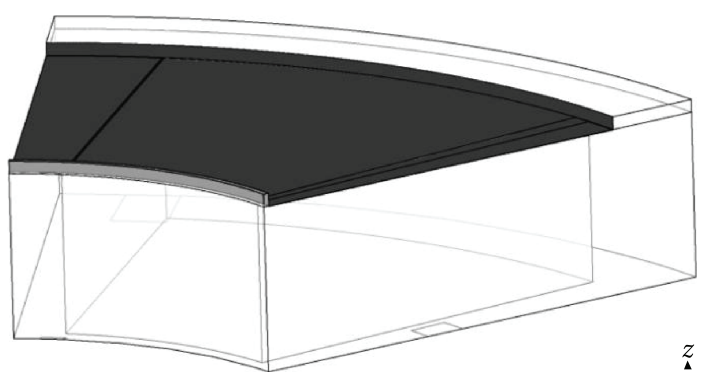

(b)

Figure 13: Two cases of collar sides immersion in oil: (a) large immersion, (b) small immersion (almost lack of immersion). 
Almost all the churning power loss is generated between pads and at the outer rim of the collar. Losses at the inner rim of collar have small contribution to overall power losses. Reduction of the churning power loss in a low-speed water turbine bearing $(v \approx 15 \mathrm{~m} / \mathrm{s})$ should result in about $10 \%$ decrease in the overall power loss and also approximately $10 \%$ gain in load carrying capacity. Because of the lack of immersion of bearing elements, that is, pads and collar, bearings should be redesigned to provide for "fail-safe" feature in the case of oil circulation system failure.

\section{Nomenclature}

\begin{tabular}{|c|c|}
\hline$D_{\text {out }}[\mathrm{m}]:$ & Outer bearing diameter \\
\hline$D_{\text {in }}[\mathrm{m}]:$ & Inner bearing diameter \\
\hline$D_{\text {ave }}[\mathrm{m}]:$ & Average bearing diameter \\
\hline$F[\mathrm{kN}]:$ & Axial load of the bearing \\
\hline$h, h_{\min }[\mu \mathrm{m}]:$ & $\begin{array}{l}\text { Film thickness, minimal film } \\
\text { thickness }\end{array}$ \\
\hline$h_{\mathrm{SG}}[\mathrm{m}]:$ & Supply groove total depth \\
\hline$h_{\text {SG_out }}[\mathrm{m}]:$ & Supply groove outlet height \\
\hline$I_{\mathrm{SG}}[\mathrm{m}]:$ & Supply groove length \\
\hline$n[\mathrm{rpm}]:$ & Rotational speed \\
\hline$P_{r}[\mathrm{~W}]:$ & Total power loss on the runner \\
\hline$P_{s}[\mathrm{~W}]:$ & Power loss on the shaft \\
\hline$P_{r \text { film }}[\mathrm{W}]:$ & Power loss in the thin film \\
\hline$P_{r \text { groove }}[\mathrm{W}]:$ & Power loss in the groove \\
\hline$P_{\text {collar_in }}[\mathrm{W}]:$ & $\begin{array}{l}\text { Power loss on the inner side (collar) } \\
\text { of the shaft }\end{array}$ \\
\hline$P_{\text {collar_out }}[\mathrm{W}]:$ & $\begin{array}{l}\text { Power loss on the outer side (collar) } \\
\text { of the shaft }\end{array}$ \\
\hline$T\left[{ }^{\circ} \mathrm{C}\right]:$ & Temperature \\
\hline$V[\mathrm{~m} / \mathrm{s}]:$ & Bearing sliding velocity \\
\hline$U=u+\bar{U}[\mathrm{~m} / \mathrm{s}]:$ & Fluid velocity \\
\hline$u[\mathrm{~m} / \mathrm{s}]:$ & Time varying component of velocity \\
\hline $\bar{U}[\mathrm{~m} / \mathrm{s}]:$ & Average component of velocity \\
\hline$h, h_{\mathrm{tot}}\left[\mathrm{m}^{2} / \mathrm{s}^{2}\right]:$ & Enthalpy, total enthalpy \\
\hline$\mu[\mathrm{Pa} \cdot \mathrm{s}]:$ & Oil viscosity \\
\hline$\lambda[\mathrm{W} / \mathrm{m} \cdot \mathrm{K}]:$ & Oil thermal conductivity \\
\hline$\rho\left[\mathrm{kg} / \mathrm{m}^{3}\right]:$ & Oil density \\
\hline$\tau[\mathrm{Pa}]:$ & Molecular stress tensor \\
\hline$S_{M}, S_{E}:$ & $\begin{array}{l}\text { External sources of momentum and } \\
\text { thermal energy. }\end{array}$ \\
\hline
\end{tabular}

\section{References}

[1] A. J. Leopard, "Tilting pad bearings: limits of operation," Lubrication Engineering, vol. 32, no. 12, pp. 637-644, 1976.

[2] C. M. Ettles, "Hot oil carry-over in thrust bearings," Proceedings of the Institution of Mechanical Engineering, vol. 184, no. 12, part 3L, pp. 75-81, 1970.

[3] F. A. Martin, "Tilting pad thrust bearings: rapid design aids," Institution of Mechanical Engineers, Tribology Convention, no. 12, paper 16, pp. 120-138, 1970.

[4] M. K. Bielec and A. J. Leopard :, "Directed lubrication for tilting-pad thrust bearings," Institution of Mechanical Engineers, Tribology Convention, vol. 184, no. 12, paper 13, pp. 93-102, 1970.
[5] N. H. New, "experimental comparison of flooded, directed, and inlet orifice type of lubrication for a tilting pad thrust bearing," Transactions of the ASME, Journal of Lubrication Technology, vol. 96, no. 1, pp. 22-27, 1974.

[6] G. Rotta, "Modeling of the gap between thrust bearing pads," Tribologia, vol. 35, no. 4, pp. 133-141, 2004 (Polish).

[7] G. Rotta and M. Wasilczuk, "CFD analysis of the lubricant flow in the supply groove of a hydrodynamic thrust bearing pad," in Proceedings of the ASME/STLE International Joint Tribology Conference, (IJTC '07), paper no. IJTC2007-44304, pp. 307-309, San Diego, Calif, USA, October 2007.

[8] M. Wasilczuk and G. Rotta, "Modeling lubricant flow between thrust-bearing pads," Tribology International, vol. 41, no. 9-10, pp. 908-913, 2008.

[9] G. Rotta, M. Wasilczuk, and L. Dabrowski, "Some aspects of using CFD in analysis of hydrodynamic lubrication," in Proceedings of 13th Nordic Tribology Symposium Nordtrib, paper no. NT2008-127-31, 2008.

[10] G. Rotta, The influence of the arrangement of the gap between pads on the performance of a thrust bearing [Ph.D. thesis], Gdansk University of Technology, 2009.

[11] L. Dabrowski and M. Wasilczuk, "Evaluation of water turbine hydrodynamic thrust bearing performance on the basis of thermoelastohydrodynamic calculations and operational data," Proceedings of the Institution of Mechanical Engineers, Part J, vol. 218, no. 5, pp. 413-421, 2004.

[12] ANSYS 11.0 documentation.

[13] J. H. Vohr, "Prediction of the operating temperature of thrust bearings," Journal of Lubrication Technology, vol. 103, no. 1, pp. 97-106, 1981.

[14] A. M. Mikula and R. S. Gregory, "Comparison of tilting pad thrust bearing lubricant supply methods," Journal of Lubrication Technology, vol. 105, no. 1, pp. 39-47, 1983.

[15] N. H. New, "Comparison of flooded and directed lubrication tilting pad thrust bearings," Tribology International, vol. 12, no. 6, pp. 251-254, 1979. 

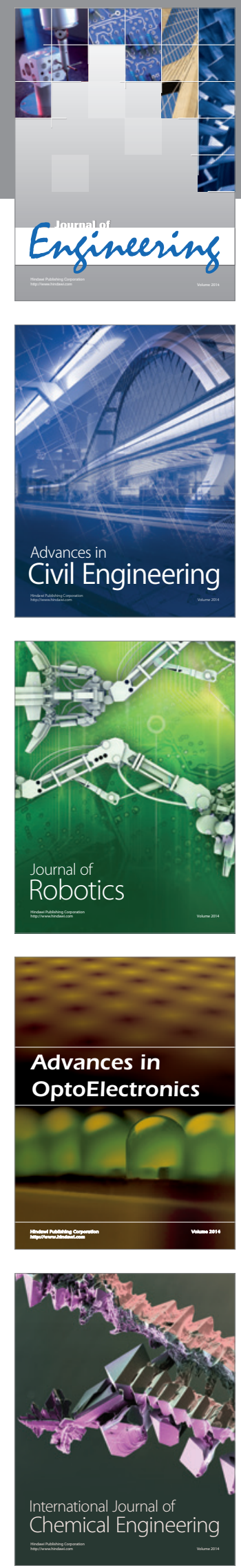

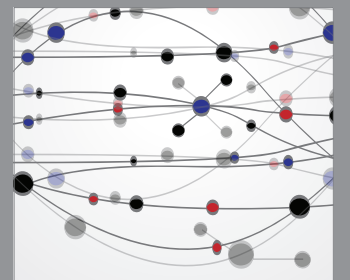

The Scientific World Journal
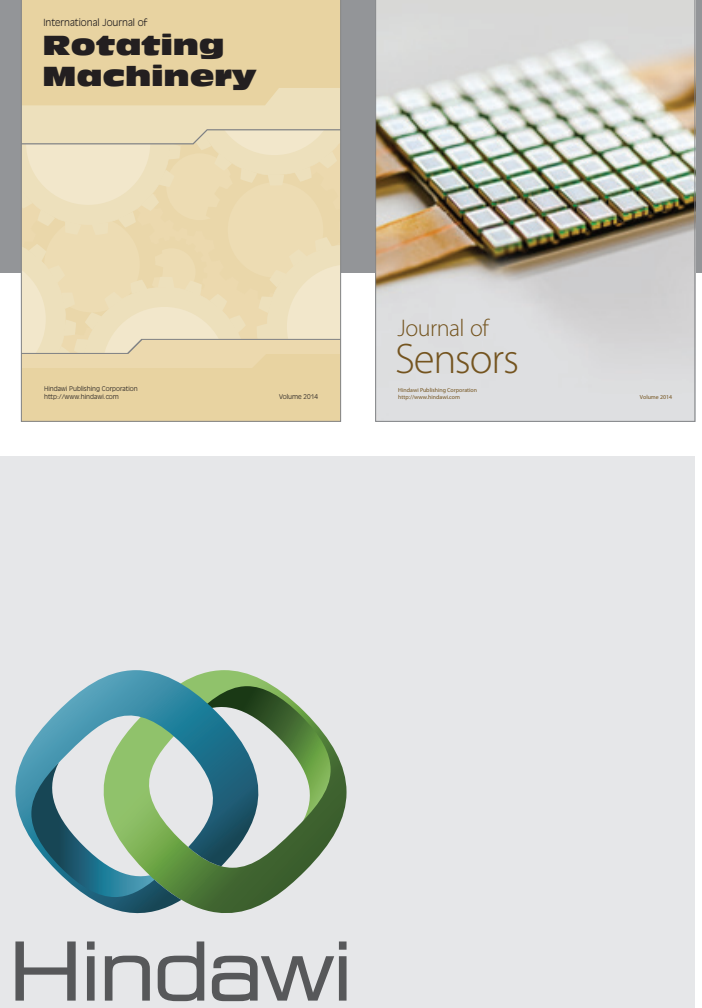

Submit your manuscripts at http://www.hindawi.com
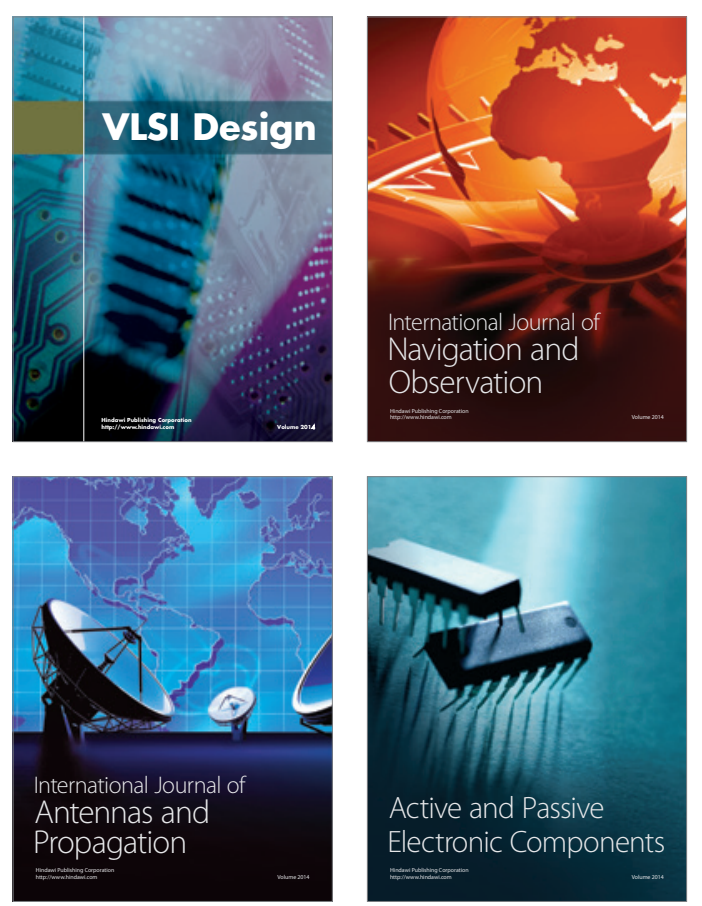
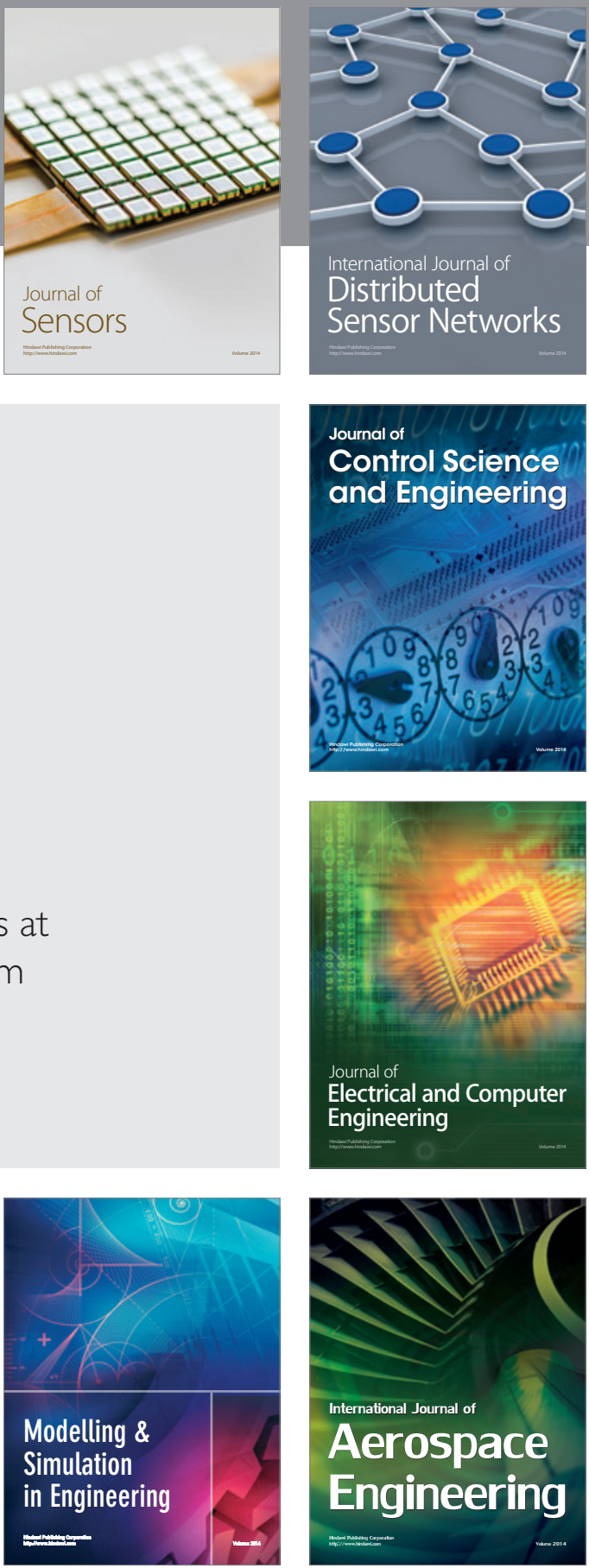

Journal of

Control Science

and Engineering
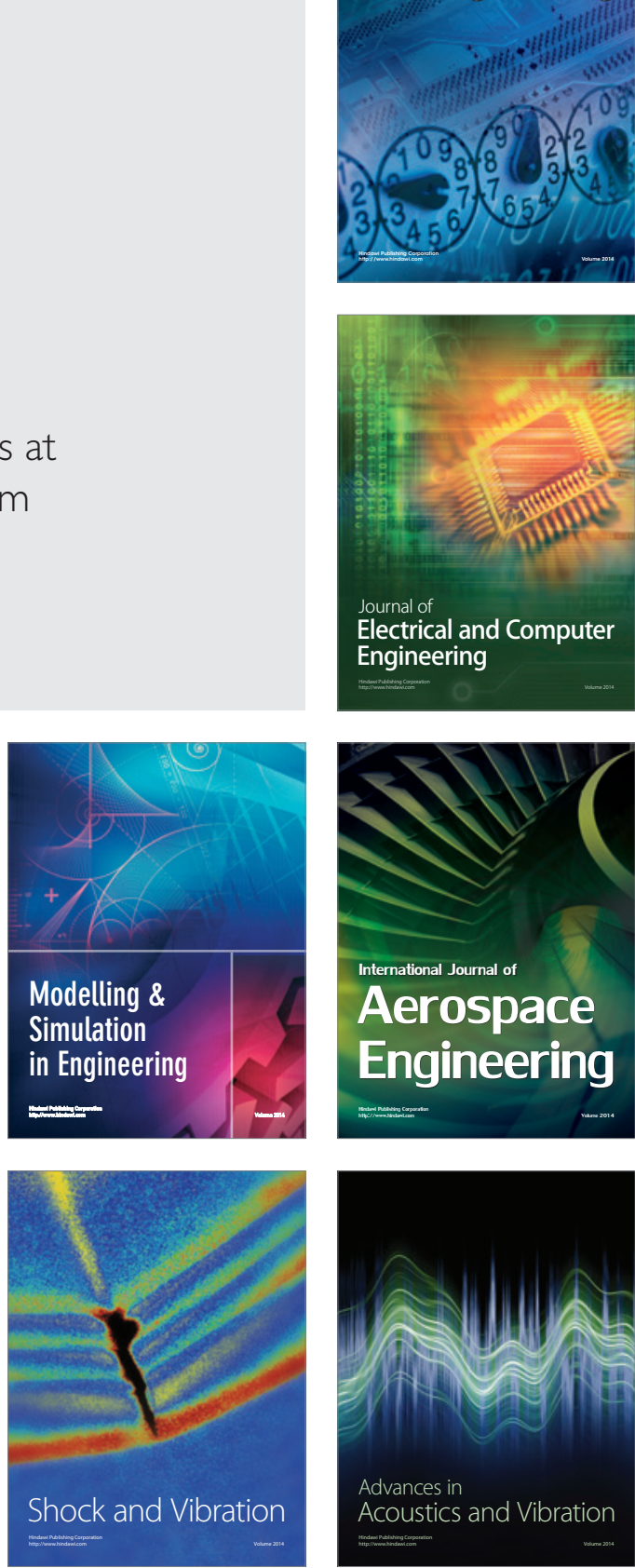